\title{
Einzelstoff-Isotopenanalyse zur Beurteilung des Abbauverhaltens von Methyl-tert-butylether (MTBE) an einem Altlastenstandort
}

Michael Berg, Luc Zwank, Jakov Bolotin, Christoph Aeppli, Andreas Häner, Matthias Möller, Christoph Munz, Urs Ziegler

\section{Zusammenfassung}

Anltand von Einzelstoffisotopenanalysen wurde an einem Benzintunfallstandort abgeklärt, ob der Benzinzusatzstoff Methy-tert-butylether (MTBE) in kontaniniertem Grundwasser abgebaut wird, oder ob es sich bei der Konzentrationsverminderung lediglich um Verdünmung und/oder Verflüchtigung im Grundwasserabstron handelt. Diese Methode erwies sidı in Vergleich zum Ansatz der Massenbilanz als äusserst aussagekräftig.

In der Schadstofffatme des Unfallstandortes Bahnhof ZürichAffoltern (Schweiz) whrden Grundwasserproben für Konzentrationsmessungen von MTBE und dessen Abbauprodukten, sowie zur Bestimunung der Isotopensignaturen $\left(\partial^{13} \mathrm{C}\right.$ und $\left.\partial^{2} H\right)$ von MTBE erhoben. Die MTBE-Konzentrationen nahmen entlang der Falme von $497 \mu \mathrm{g} / \mathrm{L}$ auf $<0.1 \mu \mathrm{g} / \mathrm{L}$ ab. Von den potentiellen Abbauprodukten war tert-Bulylalkohol (TBA) mit Konzentrationen bis zu $232 \mathrm{ug} / \mathrm{L}$ der häufigste Vertreter. In einigen Proben wurden Spuren von Aceton (maximal $22 \mu \mathrm{g} / \mathrm{L}$ ) gemessen, Isopropanol war nicht nachweisbar:

Die Isotopensignatur von MTBE änderte sich mit abnelmender Konzentration und zunehmendem Abstand vom Schadstoffherd beim Kohlenstoff nur seln geringfigig von $-29.4 \%$ bis $-29.0 \%\left(\mathrm{o}^{1.3} \mathrm{C}\right)$. Die enmittelten Anreicherungsfaktoren (c) waren mit $-0.12 \pm 0.3 \%$ ( $\left.{ }^{13} \mathrm{C}\right)$ statistisch nicht signifikant und viel kleiner als die in der Literatur beschriebenen Erfalrungswerte für aeroben und anaeroben MTBE Abban. Für Wasserstoff wurde eine elwas ausgeprägtere Anreicherung von-197\% bis $-166 \%$ ( $\left.\partial^{2} H\right)$ gentessen, woraus ein $\varepsilon$ von $-9.1 \pm 2.9 \%\left({ }^{2} \mathrm{H}\right)$ resultierte. Dieser Wert nähert sich dem einzigen bisher für Wasserstoff besclurieben $\varepsilon$ für anaeroben MTBE Abbau an (-11.4\%o). Ein anaerober MTBE Abbau hätte jedoch auch eine stark ausgeprägte Kohlenstofffraktionierung zur Folge (Literaturwerte $\varepsilon=-8.1 \pm 0.9 \%$ ), was bei Affoltern nicht der Fall ist. Der beobachtele Verlauf der Isotopensignaturen entlang der Schadstofffahne weist elser auf einen sehr geringen und ötlich stark begrenzten aeroben MTBE-Abbau mit anschliessender Verdümmung der Isotopensignaturen hin.

Aufgrund der fehlenden Hinweise anf einen signifikanten MTBE-Abbau muss die seit dem Benzinunfall von 1994 um $2-3$ Grössenordnungen verminderte MTBE Konzentration hauptsächlicli auf Evaporation und Auswaschung zurückzuführen sein, und TBA sowie Aceton kömen nicht als MTBEAbbauprodukte betrachtet werden.

\section{Einleitung}

MTBE gehört zu den organischen Chemikalien mit dem grössten Produktionsvolumen weltweit. Es wird dem Benzin vor allem zur Erhöhung der Oktanzahl aber teilweise auch als Sauerstoffquelle für eine verbesserte Verbrennung bis zu einem Anteil von $15 \% \mathrm{zu}-$ gesetzt. Aufgrund der hohen Einsatzmengen, seiner physikalisch-chemischen Eigenschaften (Wasserlöslichkeit ca, $42 \mathrm{~g} / \mathrm{L}$, hoher Dampfdruck, hohe Mobilität im Grundwasser, sehr geringe Sorption an Mineralund Huminstoffe [1]) und des langsamen biologischen Abbaus, werden Spuren von MTBE $(0.02-0.20 \mu \mathrm{g} / \mathrm{L})$ häufig in Oberflächengewässern und Grundwasser und damit auch in Trinkwasserfassungen nachgewiesen [2]. Nach einem Schadenfall kann die MTBE-Konzentration im Abstrombereich der Unfallstelle über dem Geruchs- und Geschmacksschwellenwert von 15 $-40 \mu \mathrm{g} / \mathrm{L}$ liegen. Für die Beurteilung eines Unfall-oder Altlastenstandortes ist die Identifizierung von in-situ Abbau daher wichtig. Oft stösst der Ansatz der Massenbilanz an seine Grenzen, da es sehr schwierig ist zu unterscheiden, ob eine gemessene Konzentrationsabnahme durch Abbau oder durch Verdünnung verursacht wurde.

Eine viel versprechende Methode zur Identifizierung von intrinsischem Abbau ist die Einzelstoffisotopenanalyse (Compound-specific Stable Isotope Analysis, CSIA). Dabei werden die Verhälttnisse von ausgewähl ten stabilen Isotopen einzelner Schadstoffe bzw. deren Abbauprodukte gemessen.

Die meisten chemischen Elemente besitzen stabile Isotope. So kommt zum Beispiel Kohlenstoff sowohl mit der Masse $12\left({ }^{12} \mathrm{C}\right)$ als auch mit der Masse $13\left({ }^{13} \mathrm{C}\right)$ vor. Beide Isotope sind Bestand teil aller kohlenstoffhaltigen Substanzen. Transformationsprozesse organischer Substanzen (milkrobieller Abbau, chemische Reaktionen), bei denen Bindungen gebrochen und/oder gebildet werden, verändern deren Isotopenverhältuisse. Eine Bindung zwischen schweren Isotopen ist geringfügig stabiler, so dass beim Abbau jene Bindungen mit leichteren Isotopen schneller gespalten werden. Ein Abbauprodukt hat demzufolge einen grösseren $\mathrm{An}$ teil an leichteren Isotopen als die Ausgangssubstanz. Dieser Effekt (Isotopenfraktionierung) kann an einem kontaminierten Standort genutzt werden um Hinweise zu erhalten, ob ein in-situ Abbau von Schadstoffen stattfindet. Findet man im Grundwasser mit zumehmender Distanz von der Schadstoffquelle neben einer Verminderung der Konzentrationen auch eine Veränderung der Isotopenzusammensetzung, so bedeutet dies, dass auf dem Fliessweg ein Abbau und nicht nur eine Ver- 
dünnung des Schadstoffs stattfindet. Bislang war der Einsatz solcher Isotopenuntersuchungen an organischen Einzelstoffen auf Standorte mit hohen Schadstoffkonzentrationen $(>200 \mu \mathrm{g} / \mathrm{L})$ beschränkt, da die Empfindlichkeit der bestehenden Messmethoden sehr gering war. Durch direkte Kopplung einer selektiven Anreicherungsmethode (Purge and Trap, P\&T) mit GCIRMS ist es seit kurzem möglich, die Isotopensignaturen von flüchtigen Verbindungen in tiefen Konzentrationen $(\leq 5 \mu \mathrm{g} / \mathrm{L})$ zu bestimmen [3].

\section{Unfall- und Standortbeschreibung}

Am 8. März 1994 entgleiste beim BahnhofZürich-Affoltern ein Benzintankzug. Einige der Tankwagen kippten und rissen einen Mast der $132 \mathrm{kV}$ Übertragungsleitung um. In der Folge fingen die leckgeschlagenen Kesselwagen Feuer (Abbildung 1). Unverbranntes Benzin versickerte in den Untergrund. Die beschädigten Kesselwagen enthielten rund $400 \mathrm{t}$ Benzin, wovon schätzungsweise $80 \mathrm{t}$ in den Untergrund versickerten. Als Sanierungsmassnahme wurde im März 1994 die stark benzinhaltige oberste Schicht des Untergrunds abgetragen. Um möglichst viele Benzin-Inhaltsstoffe aus dem Untergrund zu entfernen, wurde von 1994 bis 1997 an der Unfallstelle mittels vier Anlagen mit bis in $12 \mathrm{~m}$ Tiefe reichenden Saugrohren die Bodenluft abgesaugt. Die nach diesen Massnahmen im Untergrund verbleibende Benzinmenge wurde auf $c a .7 \mathrm{t}$ geschätzt, davon ca. 2 t MTBE [4]. Man hoffte, dass sich diese Menge mikrobiell abbauen würde.

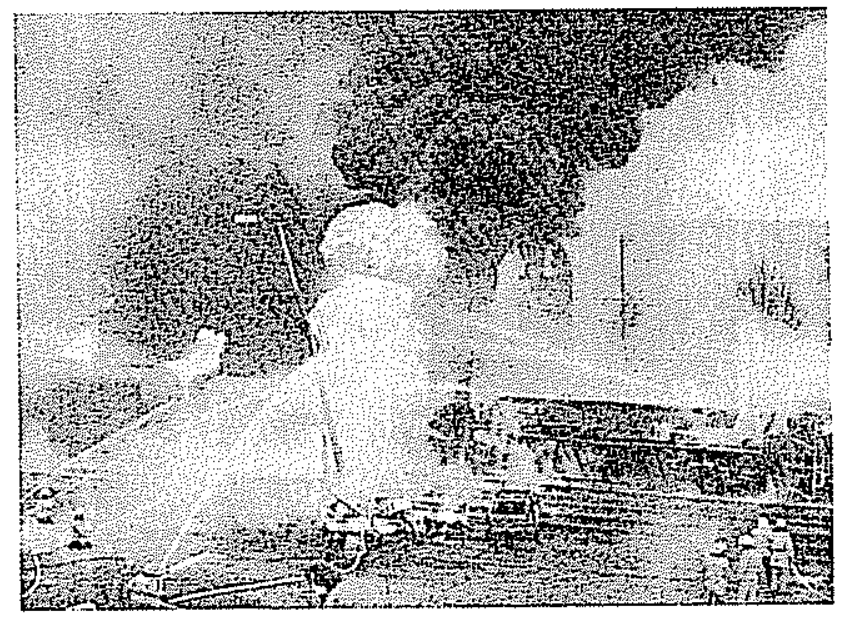

Abbildung 1: Benzinunfall am Bahnhof Zürich Affoltern 1994 (Foto: Unfalltechnischer Dienst, Züridi).

Der Untergrund am Unfallstandort besteht grösstenteils aus schlecht durchlässigem, sandig-kiesigem Moränenmaterial. Der Grundwasserspiegel befindet sich in 12 bis $15 \mathrm{~m}$ Tiefe. Im Abstrombereich befanden sich Kiesgruben, welche vor rund 70 Jahren stillgelegt und aufgeschüttet worden sind. Daraus resultiert ein heterogener Aquifer mit schlecht definierter Grundwasser-Fliessrichtung. In Abbildung 2 sind die Lage der Piezometer und die grossräumige Grundwasserfliessrichtung ersichtlich.

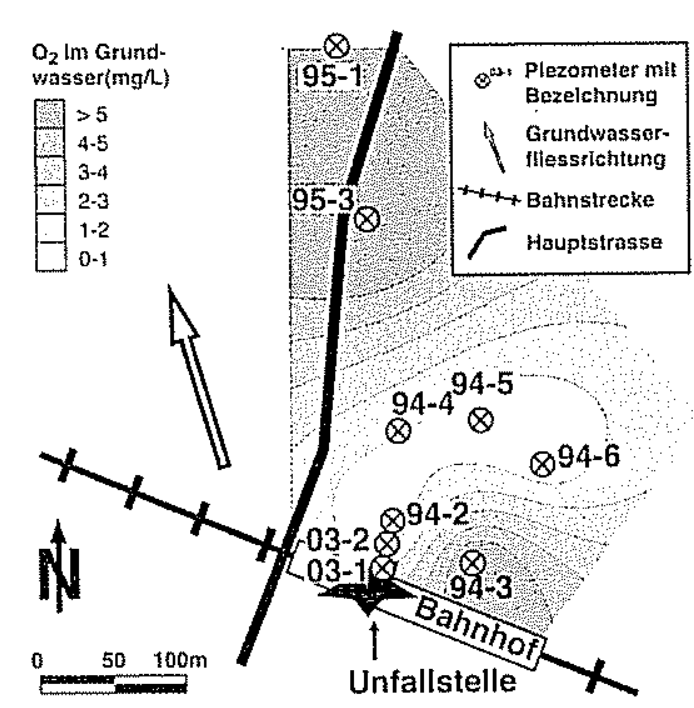

Abbildung 2:

Unfallstandort und Lage der Piezometer am 14.07. 2003. Die schattierten Fächen entsprechen dem im Grundwasser gelösten Sauterstoffgehalt (Kriging-Interpolation). Sechs der neun beprobten Piezometer liegen in Zonen mit $<2 \mathrm{mg}$ gelösten Sauerstoff pro Liter:

Die Redox- und Nährstoffparameter des Grundwassers sind in Tabelle 1 zusammengefasst. Vom Obstrom gelangt sauerstoff-, nitrat- und sulfathaltiges Grundwasser in die Kontamination. Im Bereich des Piezometers 03-1 herrschen suboxische Bedingungen. Nitrat, Mangan(IV) und Eisen(III) werden nur unwesentlich verbraucht. Im nahen Abstrombereich (Piezometer $03-2,942,94-6,94-5)$ herrschen anoxische Bedingungen. Sauerstoff ist für den biologischen Abbau nicht mehr verfügbar, Nitrat ist verbraucht, Sulfat wird abgereichert und Eisen sowie Mangan reduziert und aufgelöst. Ab Piezometer 94-4 sowie am Rand der Kontaminationsfahne (Piezometer 94-3) herrschen wieder aerobe Bedingungen. Dieser Standort dürfte bezüglich der Nährstoffe limitiert sein, da o-Phosphat an keiner Messstelle nachgewiesen werden konnte.

\section{Probenahme und Methoden}

Bei den neun Piezometern wurden am 14. Juli 2003 Grundwasserproben erhoben. Nach ausreichendem Vorpumpen (ca. 400 Liter) befullte man von jeder Messstelle drei $60 \mathrm{~mL}$. Flaschen ohne Headspace, säuerte die Proben mit $\mathrm{HCl}$ an (bis $\mathrm{pH}<2$ ) und verschloss die Flaschen mit einem Teflonseptum. Bis zur Messung wurden die Proben bei $4^{\circ} \mathrm{C}$ gelagert. Um einer Verflüchtigung der Substanzen vorzubeugen, verwendete man für jede Messserie eine zuvor ungeöffnete Flasche.

Die Konzentrationsbestimmung von MTBE, TBA, Isopropanol und Aceton erfolgte mit direkter wässriger Injektion und Gaschromatographie/Massenspelktrometrie (DAI-GC/MS) [5]. Die Verhältnisse der Kohlenstoff- und Wasserstoffisotope von MTBE erfolgten mit Gaschromatographie-Isotopenverhältnis/Massenspektromerie (GC-IRMS). Da GC-IRMS mehr als 100-fach 
Tabelle 1: Redox- und Nährstoffparmeter in Grundwasser am 14.07. 2003.

\begin{tabular}{|c|c|c|c|c|c|c|c|c|c|}
\hline I'iezometer & $\begin{array}{c}\mathrm{O}_{2} \\
\mathrm{mg} / \mathrm{t}\end{array}$ & $\begin{array}{l}\text { Nitrat } \\
\operatorname{mg~} \mathrm{NO}_{3}+\mathrm{L}\end{array}$ & $\begin{array}{l}\text { Mn gelöst } \\
\mathrm{mg} \operatorname{Mn}(\mathrm{I}) / \mathrm{L}\end{array}$ & $\begin{array}{l}\text { Fe gelöst } \\
\text { mg Fe(II)/L }\end{array}$ & $\begin{array}{c}\text { Sulfat } \\
\text { mg SO. }{ }^{2} / \mathrm{L}\end{array}$ & $\begin{array}{l}\text { o-Phosphat } \\
\mathrm{mgg} \mathrm{PO}{ }_{4}^{2} j \mathrm{~L}\end{array}$ & $\begin{array}{c}\text { Nitrit } \\
\text { mgg } \mathrm{NO}_{3} / \mathrm{L}\end{array}$ & $\begin{array}{l}\text { Ammonium } \\
\operatorname{mg} \mathrm{NH}_{4}^{+} / \mathrm{L}\end{array}$ & $\begin{array}{l}\mathrm{DOC}^{\mathrm{b}} \\
\mathrm{mg} / \mathrm{L}\end{array}$ \\
\hline $03-1$ & 1.8 & 12.7 & 0.2 & 0.6 & 35.8 & $<0.01$ & 0.09 & $<0.05$ & 1.1 \\
\hline $03-2$ & 0.7 & $<0.5$ & 0.5 & 1.6 & 30.8 & $<0.01$ & 0.07 & $<0.05$ & 1.8 \\
\hline 942 & 0.2 & $<0.5$ & 0.6 & 1.0 & 1.0 & $<0.01$ & $<0.01$ & 0.07 & 2.6 \\
\hline $94-6$ & 0.1 & $<0.5$ & 1.3 & 2.4 & 8.8 & $<0.01$ & $<0.01$ & $<0.05$ & 1.2 \\
\hline $94-5$ & 0.2 & $<0.5$ & 1.9 & 4.2 & 17.7 & $<0.01$ & $<0.01$ & $<0.05$ & 1.8 \\
\hline $94-4$ & 1.0 & 8.6 & 0.03 & $<0.02$ & 21.7 & $<0.01$ & $<0.01$ & $<0.05$ & 0.7 \\
\hline $95-3$ & 7.6 & 19.5 & $<0.02$ & $<0.02$ & 22.3 & $<0.01$ & $<0.01$ & $<0.05$ & 0.6 \\
\hline $95-1$ & 5.5 & 16.6 & $<0.02$ & 0.02 & 15.0 & $<0.01$ & $<0.01$ & $<0.05$ & 0.6 \\
\hline $94-3$ & 6.7 & 14.3 & $<0.02$ & $<0.02$ & 31.6 & $<0.01$ & $<0.01$ & $<0.05$ & $<0.5$ \\
\hline
\end{tabular}

a nach zunehmender Entfernung zum Schadstofherd geordnet; das letzte Piezometer befindet sich am Rand der Kontamination, b gelöster organisciner Kohlenstoff.

weniger empfindlich ist als DAI-GC/MS, mussten die Substanzen für GC-IRMS Messungen mittels on-line gekoppelter Purge-and-Trap (P\&T) Anreicherung aufkonzentriert werden [3].

\subsection{DAl-GC/MS}

Die DAI-GC/MS Messungen erfolgten mit einem GC/ MS-System von Fisons (GC8000, MDs00). $1 \mu \mathrm{L}$ Grundwasserprobe wurde im On-column Injektor auf eine $60 \mathrm{~m} \times 0,32 \mathrm{~mm}$ Kapillarsäule (Stabilwax, Filmdicke $2 \mu \mathrm{m}$, Restek) mit deaktivierter Fused Silica-Vorsäule ( $8 \mathrm{~m} \times 0,53 \mathrm{~mm}$ ) injiziert. Die gaschromatographischen Parameter sind in Referenz [5] dokumentiert. Detektient wurde mittels Elektronenstoss-Ionisation (EI) und Single Ion Monitoring (SIM). Die eindeutige Identifizierung und Quantifizierung erfolgte durch jeweils zwei substanzspezifische Massen sowie deren Verhältnis, das mit dem entsprechenden Wert des Kalibrier-Standards mit einer Abweichung von $<10 \%$ übereinstimmen musste [5].

\subsection{P\&T-GC-IRMS}

$25 \mathrm{~mL}$ Grundwasserprobe wurden in einer P\&T Apparatur (Tekmar) 60 Minuten lang mit $40 \mathrm{~mL} / \mathrm{min}$ Stickstoff ausgeblasen (purge) und die Analyten auf einer VOCARB 3000 Säule (Supelco) bei $23^{\circ} \mathrm{C}$ absorbiert (trap). Anschliessend wurden die Analyten in Stickstoffstrom bei $250^{\circ} \mathrm{C}$ von der VOCARB Säule thermodesorbiert, zum Gaschromatographen überführt und in einer mit flüssigem Stickstoff betriebenen KryofokussierungsEinheit bei $-120^{\circ} \mathrm{C}$ am Kopf der GC-Trennsäule aufkonzentriert. Die Substanzen wurden mit den in Referenz [5] beschriebenen Bedingungen mit einem Trace GC (Fisons) auf einer Petrocol DH 150 Säule (150 m, Filmdicke $1 \mu \mathrm{m}$, Heliumvordruck $200 \mathrm{k} \mathrm{Pa}$ ) aufgetrennt und in einer Durchfluss-Verbrennungseinheit in $\mathrm{CO}_{2}(940$ ${ }^{\circ} \mathrm{C}$ ), respektive in $\mathrm{H}_{2}$ (Pyrolyse bei $1400^{\circ} \mathrm{C}$ ) überfüht. Nach der Trocknung der entstandenen Gase wurden die Isotopensignaturen schliesslich im Isotopenverhältnis-Massenspektromer (IRMS, DeltaPLUS-XL, Thermo Finnigan MAT) bestimmt (siehe Abbildungen 4 und 5). Eine wichtige Voraussetzung für die Ermittlung von präzisen Isotopenverhältnissen ist eine gute gaschromatographische Trennung der einzelnen Substanzen (Peaks), da dem IRMS lediglich $\mathrm{CO}_{2}$ oder $\mathrm{H}_{2}$ zugeführt wird, welches anschliessend nicht mehr von verschiedenen Substanzen unterschieden werden kann (siehe Abbildung 4).

\subsection{Delta $(\partial)$ Notation und Rayleigh-Gleichung}

Die isotopische Zusammensetzung einer Substanz wird üblicherweise auf einen internationalen Standard normiert. Aufgrund der sehr kleinen Isotopenverschiebungen ( $\%$ Bereich) gibt man die Isotopenverhälnisse in der Delta Notation mit der Einheit Promill (\%) an (Gleichung 1).

$\partial^{13} \mathrm{C}=\left(\left(\mathrm{R}_{\text {Prove }}\right) /\left(\mathrm{R}_{\text {Referenz }}\right)-1\right) \cdot 1000[\% \Theta]$

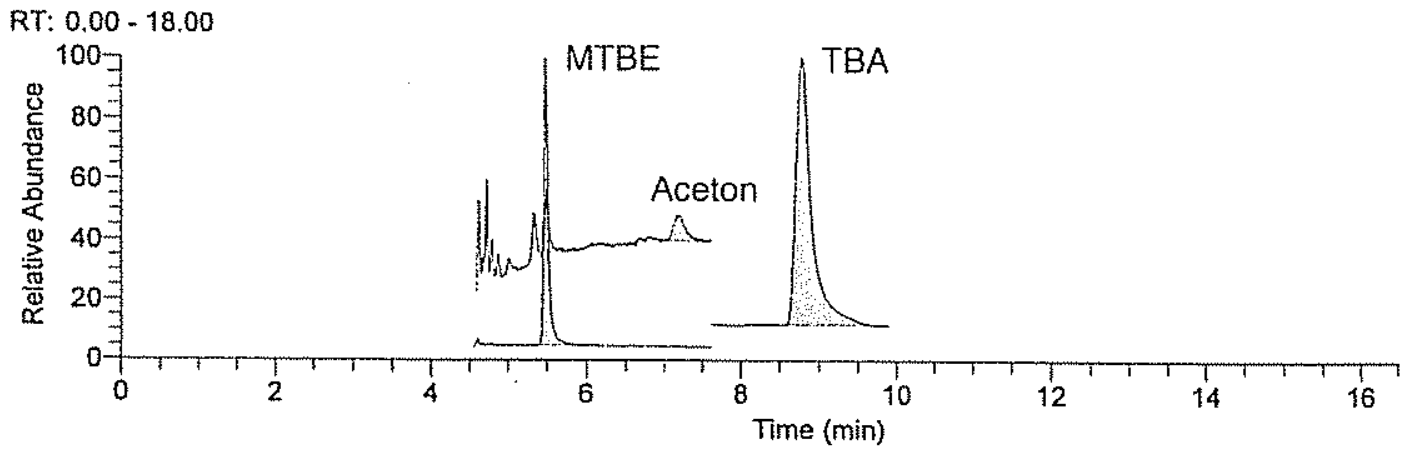

Abbildung 3: DAI-GC/MS Chromatogramm (Probe 03-1) mit den Massenspuren fïr MTBE (m/z 73), TBA (m/z 59), and ACeton $(m / z 58)$. 
Abbildung 4: Apparatives Schema der on-line gekoppelten PET-GC-IRMS Analytik (Kohlen. stoffmodus).

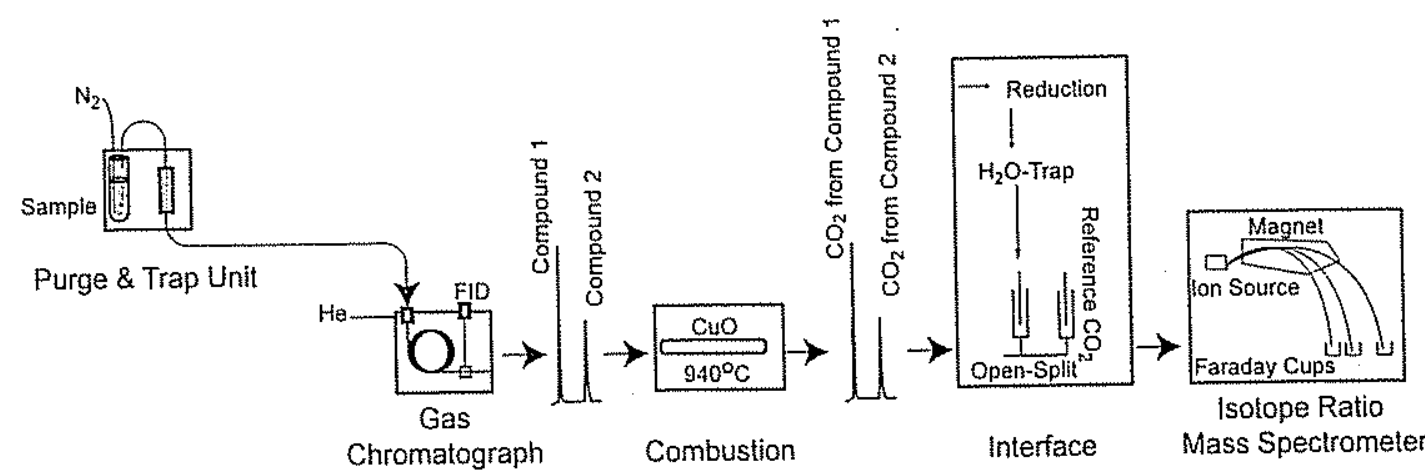

wobei $R_{\text {probe }}$ und $R_{\text {Referenz }}$ den Verhältnissen des schwereren Isotops zum leichten Isotop entsprechen (z.B. ${ }^{13} \mathrm{C}^{12} \mathrm{C}$ oder $\left.{ }^{2} \mathrm{H} /{ }^{1} \mathrm{H}\right)$. Die Normierung auf einen internationalen Standard vereinfacht den Vergleich von Messwerten, unabhängig von der Signatur des verwendeten Eichgases. Eine Substanz mit einem negativen $\partial^{13} \mathrm{C}$ Wert enthält demzufolge weniger ${ }^{13} \mathrm{C}$ als der internationale Standard.

Die Isotopenfraktionierung einer Substanz kann in einer Schadstoffahne durch den Fraktionierungsfaktor $\alpha$ oder den Anreicherungsfaktor $\varepsilon$ beziffert werden, der sich aus der von Lord Rayleigh entwickelten Gleichung zur Beschreibung einer fraktionierten Destillation ableiten lässt. Detaillierte Angaben zur Herleitung sowie zu Limitierungen dieser Gleichung sind in $[6]$ aufgeführt. In Gleichung 2 ist die RayleighGleichung am Beispiel der ${ }^{2} \mathrm{H}$ Fraktionierung wiedergegeben.

$$
\begin{array}{r}
\ln \left(\left(\partial^{2} \mathrm{H}_{\mathrm{x}}+1000\right) /\left(\partial^{2} \mathrm{H}_{\mathrm{o}}+1000\right)\right)= \\
\varepsilon / 1000^{*} \ln f=(\alpha-1) * \ln f
\end{array}
$$

wobei $\partial^{2} \mathrm{H}_{\mathrm{x}}$ und $\partial^{2} \mathrm{H}_{0}$ den Isotopenverhältnissen der untersuchten Substanz im Grundwasser am Standort $x$ und im Schadstoffherd (o) entspricht. Der verblei- bende Anteil $f$ der Substanz ist durch das Verhältnis der Konzentration (Kon $\left.z_{x} / K_{0 n z_{0}}\right)$ gegeben.

\section{Resultate und Diskussion}

4.1. Konzentrationen von MTBE und potentiellen Abbauprodukten

Unter oxischen und anoxischen Bedingungen ist TBA das primäre Abbauprodukt von MTBE. In der Literatur werden unter anderem auch Isopropanol und Aceton als produkte eines weitergehenden Abbaus genannt [1].

Die mit DAI-GC/MS gemessenen Konzentrationen sind in Tabelle 2 aufgelistet. Mit zunehmendem Abstand vom Schadstoffherd nahm die MTBE Konzentration von $497 \mu g / L$ auf Werte unter der Nachweisgrenze von $1.2 \mu \mathrm{g} / \mathrm{L}$ ab (siehe Abbildung 6). Seit dem Benzinunfall von 1994 wurde die MTBE Konzentration am Piezometer 942 von $123 \mathrm{mg} / \mathrm{L}$ auf $180 \mu \mathrm{g} / \mathrm{L}$ um einen Faktor 680 vermindert, beim Standort 946 von $33 \mathrm{mg} / \mathrm{L}$ auf $87 \mu \mathrm{g} / \mathrm{L}$ um einen Faktor 380 . Der häufigste Vertreter der potentiellen Abbauprodukte war tert-Butylalkohol (TBA) mit Konzentrationen bis zu $232 \mathrm{\mu g} / \mathrm{L}$. In drei Proben wurden Spuren von Aceton $(3.0-22 \mu \mathrm{g} / \mathrm{L})$ gemessen, Isopropanol konnte in keiner Probe nachgewiesen werden.
Abbildung 5:

Beispiel eines GC-IRMS Chromatogramms für die $\partial^{\mathrm{I3}} \mathrm{C}$ Bestimmung von MTBE. Im unteren Teil sind die Massenspuren $m / z=44,45$ und 46 ersichtlich, im oberen Teil das Massenverhältnis $45 / 44$.

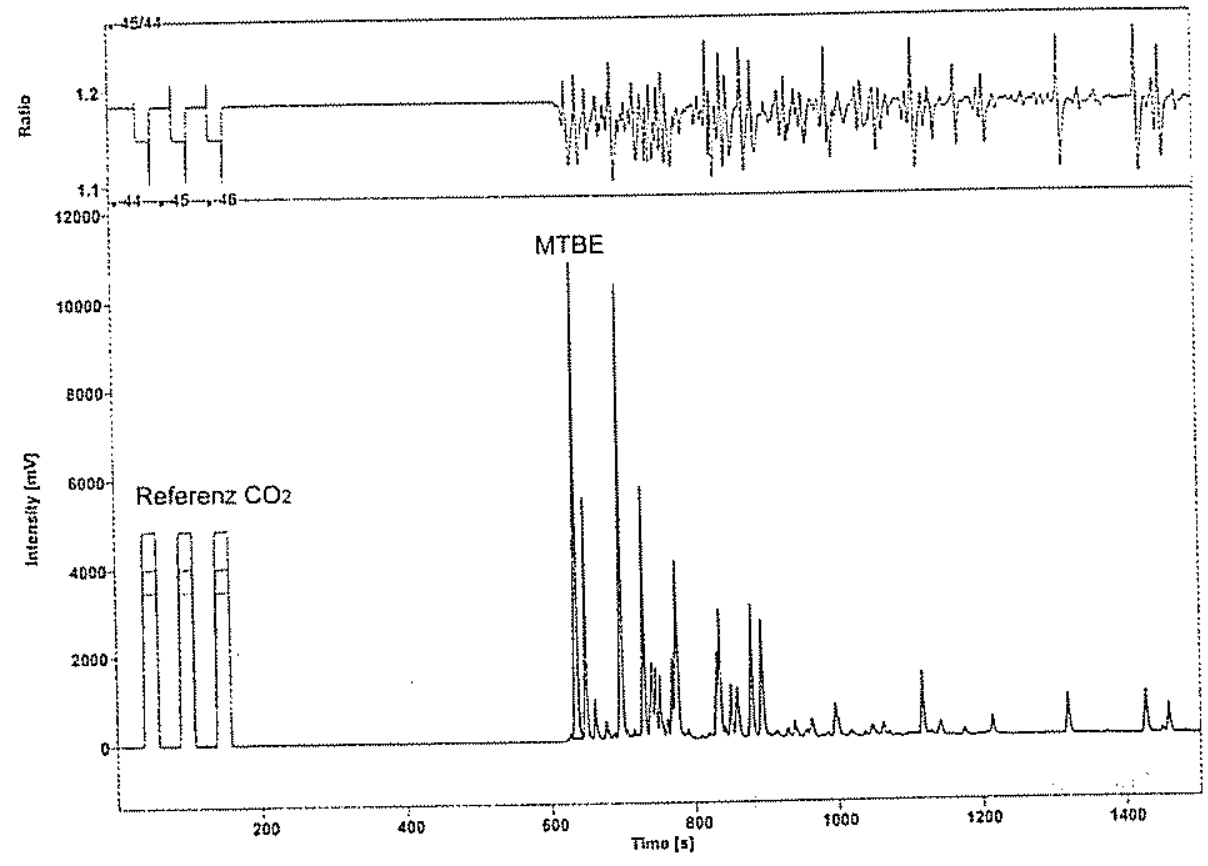


Tabelle 2; Konzentutionen der quantifizierten Substanzen

\begin{tabular}{cccccc}
\hline Piezometer & $\begin{array}{c}\text { MTBE } \\
\mu g / L\end{array}$ & $\begin{array}{c}\text { MTBE } \\
\mu g / L\end{array}$ & $\begin{array}{c}\mathrm{TBA}^{3} \\
\mu \mathrm{L}\end{array}$ & $\begin{array}{c}\text { Isopropanol }^{\mathrm{a}} \\
\mu \mathrm{g} / \mathrm{L}\end{array}$ & $\begin{array}{c}\text { Aceton }^{*} \\
\mu g / \mathrm{L}\end{array}$ \\
\hline $03-1$ & 125 & 119 & 116 & $<2.5$ & 3.0 \\
$03-2$ & 497 & 491 & 232 & $<2.5$ & 22.0 \\
$94-2$ & 180 & 170 & 98 & $<2.5$ & $<1.6$ \\
$94-6$ & 87 & 80 & 26 & $<2.5$ & 6.4 \\
$94-5$ & 15 & 12 & $<1.6$ & $<2.5$ & $<1.6$ \\
$94-4$ & 5.9 & 5.6 & $<1.6$ & $<2.5$ & $<1.6$ \\
$95-1$ & $<1.2$ & 0.13 & $<1.6$ & $<2.5$ & $<1.6$ \\
$95-3$ & $<1.2$ & $<0.1$ & $<1.6$ & $<2.5$ & $<1.6$ \\
$94-3$ & $<1.2$ & 0.26 & $<1.6$ & $<2.5$ & $<1.6$ \\
\hline
\end{tabular}

a Quantifizierung nit DAI-GC/MS (Messunsicherheit $=10 \%$ ). Konzentrationen unterhalb der Nachweísgrenze sind mit ${ }_{n}<*$ bezeichnet.

b Quantinzierung mit P\&I-GC/MS im Rahmen des üblichen Grundwassemonitorings.

\section{2. $\partial^{13} \mathrm{C}$ und $\partial^{2} \mathrm{H}$ Werte von MTBE}

Die mit einer Vielzahl von Kohlenwasserstoffen verschmutzten Grundwasserproben verursachten bei den GC-IRMS Messungen Schwierigkeiten, da die MTBE und TBA Peaks im Chromatogramm von anderen Substanzen überlagert waren. Im Unterschied zur GC/MS Analytik können überlagerte Peaks nicht anhand von substanzspezifischen Massenfragmenten isoliert werden, da einem IRMS ausschliesslich die Verbrennungs- resp. Pyrolysegase $\left(\mathrm{CO}_{2}\right.$ oder $\left.\mathrm{H}_{2}\right)$ der entsprechenden Substanzpeaks zugeführt werden. Durch die Verwendung einer $150 \mathrm{~m}$ langen Säule (Petrocol DH 150) gelang es, MTBE weitgehend von anderen Peaks zu trennen. Die für Isotopenmessungen notwendige Isolierung des polaren TBA war hingegen mit der apolaren Petrocol Säule nicht möglich.

Die gemessenen Isotopenwerte von MTBE sind in Tabelle 3 zusammengestellt. Es ist ersichtlich, dass sich die $\partial^{13} \mathrm{C}$ Werte mit zunehmender Distanz vom

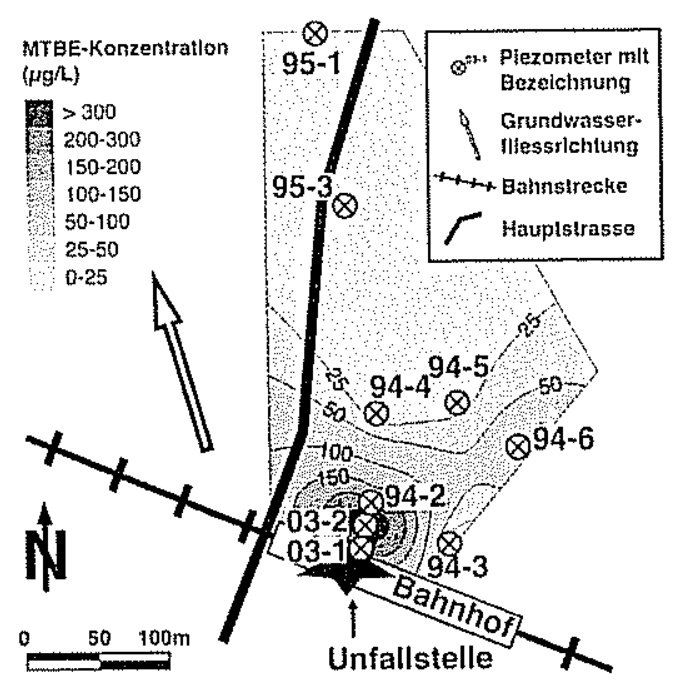

Abbildung 6:

MTBE-Konzentrationen ant Unfallstandort am 14. 07. 2003. Die schaltierten Fächen sind mit Krigging-Interpolation berechnet.
Schadstoffherd praktisch nicht verändern. Die relative Änderung der H-Isotopensignaturen ist grösser als für Kohlenstoff, selbst wenn man die höheren Standardabweichungen berücksichtig die bei $\partial^{2} \mathrm{H}$ Messungen üblich sind. In den Proben 94-3, 95-1 und 95-3 waren die MTBE Konzentrationen zu gering, um Isotopenverhältnisse bestimmen zu können.

\subsection{Anreicherung von ${ }^{13} \mathrm{C}$ und ${ }^{2} \mathrm{H}$ entlang der Schadstofffahne}

Der Anreicherungsfaktor $\varepsilon$ (siehe Gleichung 2) ist ein wichtiges Mass zur Quantifizierung des Fraktionierungsgrades einer Schadstofftransformation. Für den aeroben und anaeroben Abbau von MTBE werden in der Literatur deutlich unterscheidbare Anreicherungsfaktoren $\varepsilon$ angegeben (siehe Tabelle 4). Charakteristisch ist insbesondere, dass die $\varepsilon$-Werte im aeroben Fall für ${ }^{13} \mathrm{C}$ klein und für ${ }^{2} \mathrm{H}$ gross sind, während sich dies beim anaeroben Abbau genau umgekehrt verhält: [7].

Die Isotopen- und Konzentrationsdaten wurden gemäss der Rayleigh-Gleichung gegeneinander aufgetragen und die Anreichungsfaktoren $\varepsilon$ für Wasserstoff und Kohlenstoff bestimmt (Abbildungen 7 und 8). Der Wasserstoff-Anreicherungsfaktor von $-9.1 \pm 2.9 \%$ lässt einen anaeroben Abbau vermuten. Für Kohlenstoff ergibt sich jedoch ein ausgesprochen kleiner und statistisch nicht signifikanter $\varepsilon$-Wert von $-0.12 \pm 0.3 \%$, der sich vom anaeroben Literaturwert von $-8.1 \pm 0.9 \%$ deutlich unterscheidet.

Tobelle 4: Literaturwerte fïr Anteicherungsfaktoren $\varepsilon$ beim MTBE Abbau

\begin{tabular}{llll}
\hline MTBE Abbau & $\varepsilon^{13} \mathrm{C}$ & $\varepsilon^{2 \mathrm{H}}$ & Referenzen \\
& $\%$ & $\%$ & \\
\hline aerob & -1.75 & & {$[8],[9]$} \\
aerob & & 29 bis -66 & {$[10]$} \\
anaerob & -8.1 & & {$[8]$} \\
anaerob & & -11.4 & {$[11]$} \\
\hline
\end{tabular}


Tabelle 3:

$\delta^{13} \mathrm{C}$ und $\delta^{12} \mathrm{H}$ Werte von MTBE (Mittelwerte von 3 fach Bestimmungen)

\begin{tabular}{|c|c|c|c|c|c|c|}
\hline \multirow[t]{2}{*}{ Piezometer } & \multicolumn{2}{|c|}{ Konzentration } & \multicolumn{2}{|c|}{$\partial^{13} \mathrm{C}$ (vs VPDB) } & \multicolumn{2}{|c|}{$\partial^{2} \mathrm{H}$ (VS VSMOW) } \\
\hline & $\mu g / \mathrm{L}$ & $\pm^{\mathrm{c}}$ & $\%$ & $\pm d$ & $\%$ & $\pm d$ \\
\hline $03-1$ & 125 & 13 & -29.4 & 0.3 & -186 & 36 \\
\hline $03-2$ & 497 & 50 & -29.4 & 0.3 & -197 & 9 \\
\hline $94-2$ & 180 & 18 & -29.5 & 0.7 & -167 & 10 \\
\hline $94-6$ & 87 & 9 & -30.1 & 1.3 & -189 & 16 \\
\hline $94-5$ & 15 & 2 & -28.2 & 0.3 & -173 & 18 \\
\hline $94-4$ & 5.9 & 1 & -29.0 & 0.5 & -166 & 45 \\
\hline $95 \cdot 3$ & $<1.2$ & & & & & \\
\hline 95-1 & $<1.2$ & & & & & \\
\hline $94-3$ & $<1.2$ & & & & & \\
\hline Mittelwert & & & -29.3 & & -178 & \\
\hline Standardabweichung & & & 0.65 & & 13 & \\
\hline
\end{tabular}

a Werte für $\delta^{13} \mathrm{C}$ normiert auf internationalen Standard "Vienna Pee Dee Belemnite" (VPDB).

b Werte für $\delta^{2} \mathrm{H}$ normiert auf internationalen Standard "Vientia Standard Mean Ocean Water" (VSMOW).

c Messunsicherheit $=10 \%$.

d Standardabweichung von 3-fach Bestimmungen.

Über die (den Isotopenmessungen zugängliche) Aus* breitung der Schadstoffahne beim Bahnhof Affoltern geben die $\delta^{13} \mathrm{C}$-Daten keinen Hinweis auf einen MTBEAbbau, weder im suboxischen (Piezometer 03-1) noch im anoxischen Milieu. Da bei einem aeroben Abbau von MTBE wesentlich höhere Wasserstoffraktionierungen und relativ geringe Kohlenstoffraktionierungen auftreten (Tabelle 4), können die beobachteten $\varepsilon$-Werte im besten Fall auf einen örtlich sehr begrenzten und geringen aeroben MTBE-Abbau zurückzuführen sein. In solchen aeroben "Zellen" würden starke Wasserstoffverschiebungen und geringe Kohlenstofffraktionierungen auftreten. Durch den Weitertransport und das Vermischen mit MTBE aus Regionen des Aquifers, in welchem kein Abbau stattfindet, wird

\section{Rayleigh Plot für $\delta^{2} H$ von MTBE}

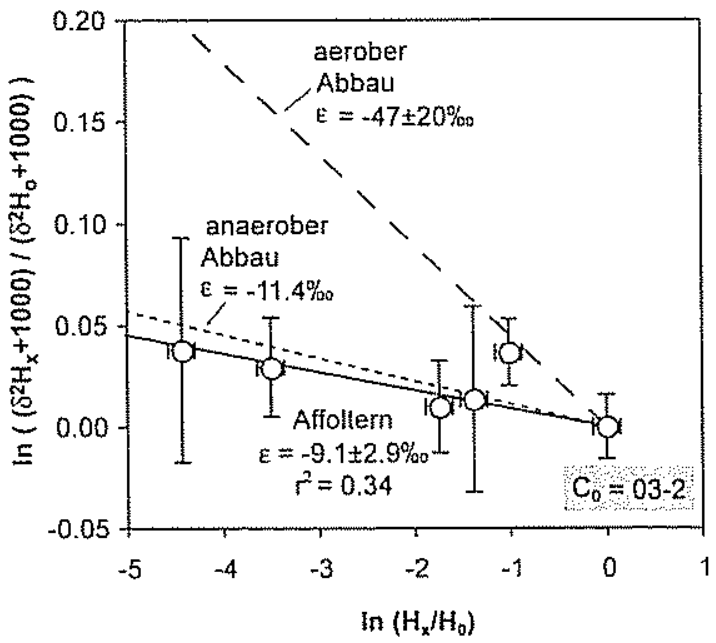

Abbildung 7:

Rayleight-Plot für $\delta^{2} H$ von MTBE, ausgehend von der höchsten MTBE Konzentration $\left(C_{o}\right)$ in der Probe 03-2. diese Isotopenanreicherung wieder „verdünnt“. Diese Situation würde die Anwesenheit einer signifikanten Wasserstofffraktionierung zusammen mit einer schwachen Anreicherung von ${ }^{13} \mathrm{C}$ erklären.

Die Konzentrationsabnahme von MTBE um mehrere Grössenordnungen im Vergleich mit den seit dem Benzinunfall von 1994 gemessenen Höchstkonzentrationen (Faktor 680 und 380 in Piezometer 94-2 bzw. 94-6; Faktor $>1000$ in den übrigen Piezometer) muss demzufolge grösstenteils auf Evaporation und Auswaschung zurückzuführen sein, und TBA sowie Aceton können nicht als MTBE-Abbauprodukte betrachtet werden. Wie in Kapitel 1 erwähnt, sorbiert MTBE äusserst schwach an Mineral- und Huminstoffe [1] und ist somit im Grundwasser sehr mobil. Weil Benzin bis zu 1\% TBA

\section{Rayleigh Plot für $\delta^{13} \mathrm{C}$ von $\mathrm{MTBE}$}

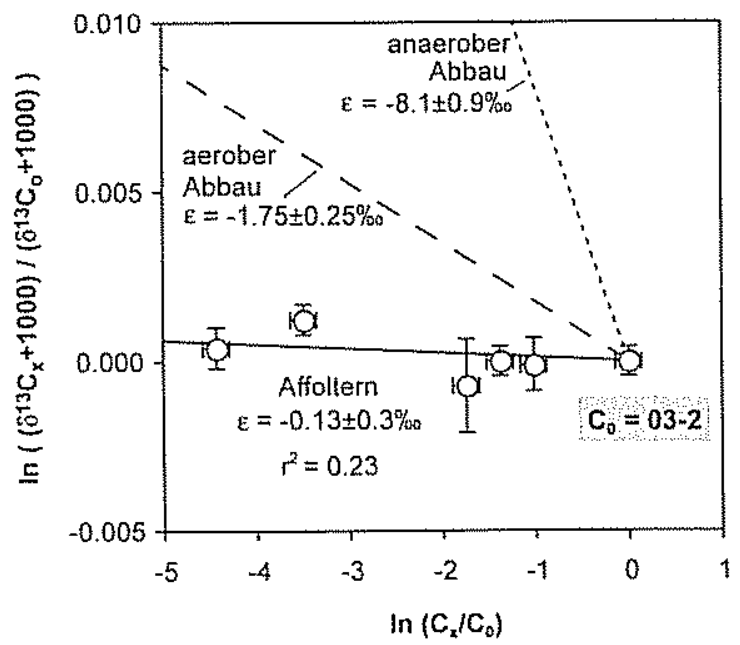

Albildung 8:

Rayleggli-Plot fiir $\delta^{13} \mathrm{C}$ von MTBE, ausgehend von der höchsten MTBE Konzentration $\left(C_{n}\right)$ in der Probe 03-2. 
enthalten kann [1], wird vermutet, dass das schlecht abbaubare TBA bereits als Verunreinigung im versickerten Benzin vorhanden war und dass höchstens ein geringfügiger Anteil aus der Transformation von MTBE stammt. Unterstützt wird diese Vermutung durch zwei Übersichtsanalysen vom Juli und August 1994, in denen TBA in signifikanten Konzentiationen in den $\mathrm{Pi}$ ezometern 94-2 und 94-4 gemessen wurde. Allerdings erfolgten die GC-Analysen durch blossen Vergleich der Retentionszeiten, so dass die Daten nicht als gesichert gelten können. Der im Vergleich zu MTBE relativ hohe Anteil an TBA ist wahrscheinlich auf die nach dem Unfall eingeleitete Bodenluftabsaugung zurückzuführen, bei der die Konzentration des gutfüchtigen MTBE (log $\mathrm{K}_{\mathrm{fl}}-3.23$ atm ${ }^{*} \mathrm{~m}^{3} / \mathrm{mol}$ ) viel stärker vermindert wurde als diejenige von TBA $\left(\log \mathrm{K}_{\mathrm{H}}-4.85 \mathrm{~atm}{ }^{*} \mathrm{~m}^{3} / \mathrm{mol}\right)$. Das sehr gut abbaubare Aceton entsteht beim anaeroben Abbau einer Vielzahl von Kohlenwasserstoffen und muss seinen Ursprung bei anderen Vorläufersubstanzen liaben.

\section{Fazit}

Die Untersuchungen mit Isotopensignaturen am Benzin-Unfallstandort Bahnhof Zürich-Affoltern zeigen, dass MTBE insbesondere in der Zone mit stark reduzierenden Bedingungen biologisch nicht abgebaut wird. In diesen Bereichen wurden auch 9 Jahre nach dem Unfall signifikante, wenn auch gegenüber den ursprünglichen Werten deutlich tiefere, Konzentrationen an Benzinkohlenwasserstoffen gemessen.

Zwischen 100 und $250 \mathrm{~m}$ unterhalb der Unfallstelle sind die Konzentrationen von MTBE sowie weiterer Schadstoffe unter der Bestimmungsgrenze von $0.1 \mu \mathrm{g} /$ L. In den Proben dieser aeroben Zone (Piezometer 953 und 95-1) konnten daher die Isotopensignaturen von MTBE nicht bestimmt werden. Ein aerober Abbau von MTBE ist in dieser Zone jedoch nicht vollständig ausgeschlossen.

Generell zeigen die Untersuchungen, dass die Methode der Einzelstoffisotopenanalyse gut geeignet ist, das Abbativerhalten von organischen Schadstoffen zu untersuchen. Diese Information ist für die Beur-

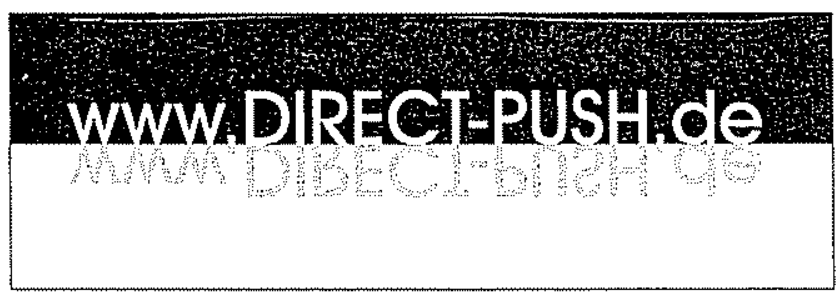

\section{Anzeigenannahmeschluss} für das Heft 2/2005 von altlasten spelktrum ist der 13. März 2005

Tel $030 / 250085-850-\mathrm{Fax} 030 / 250085-870$ QMail W.Plochl@ESVmediende teilung des natürlichen Selbstreinigungspotenzials eines Altiastenstandortes essentiell.

\section{Danksagung}

Diese Studie wurde im Auftrag des Schweizerischen Bundesant für Unwelt, Wald und Landschaft (BUWAL) durchgeführt. Die Autoren danken Christoph Wenger für die Initiierung und Begleitung dieser Arbeit sowie den Schweizerischen Bundesbahnen (SBB) für die Bereitstellung der standortrelevanten Daten früherer Untersuchungen.

\section{Referenzen}

(1) Sclamidt TC, Schirmer M, Weiss H, Haderlein SB (2004) Microbial degradation of methyl tert-butyl ether and tert-butyl alcohol in the subsurface, Journal of Contaminant Hydrology 70 , p. 173-203.

[2] Schmidt TC, Duong HA, Berg M, Haderlein SB (2001) Analysis of Fuel Oxygenates in the Enviromment: Critical Review, Analyst $126, p, 405-413$.

(3) Zwank L, Berg M, Schmidt TC, Haderlein SB (2003) CompoundSpecific Carbon Isotope Analysis of Volatile Organic Compounds in the Low-Microgram per Liter Range, Analytical Chemistry 75, p. 5575-5583.

[4] Munz C, Gälli R, Kiayias G, Schmid B (1998) Risk Assessment, Mass Balance and Successful Remediation of a Major Gasoline Spill in a Residential Area. In Harder, W., Arendt, F., Hart, I. (eds.). Contaminated Soil 98 , Thomas Telford Publishing, London. p. 1067-1068.

[5] Zwank L. Schmidt TC, Haderiein SB, Berg M (2002) Simultaneous Determination of Fuel Oxygenates and BTEX Using Direct Aqueous Injection Gas Chromatography Mass Spectrometry (DAI-GC/MS), Environmental Science \& Technology 36, p. 20542059.

[6] Schmidt TC, Zwank L, Elsner M, Berg M, Meckenstock RU, Haderlein SB (2004) Compound-specific stable isotope analysis of organic contaminants in natural enviromments: a critical review of the state of the art, prospects, and future challenges, Analytical and Bioanalytical Chemistry 378, p. 283-300.

(7) Zwank L, Berg M. Schmiclt TC. Schwatzenbach RP, Haderlein SB (2004) ldentification of in situ MTBE Degradation Pathways in a Contaminated Aquifer Using Carbon and Hydrogen Compound-Specific Stable Isotope Analysis, Environmental Science \& Tecinology $39(4)$.

[8] Gray IR, Lachampe-Couloume G, Glandi D, Scow KM, Wilson RD, Mackay DM, Sherwood Lollar B (2002) Carbon and Hydrogen Isotopic Fractionation during Biodegradation of Methyl tert-Butyl Ether, Environmental Science \& Technology 36, p. 1931-1938.

(9) Hunkeler D, Butler BJ, Aravena R, Barker JF (2001) Monitoring Biodegradation of Methyl tert-Butyl Ether (MTBE) Using Compound-Specific Carbon Isotope Analysis, Environmental Science \& Technology 35, p. 676-681.

110] Kolhatkar R, Kuder T, Philp P. Allan J, Wilson ]T (2002) Use of Compound-Specific Stable Carbon Isotope Analyses To Demonstrate Anaerobic Biodegradation of MTBE in Groundwater at a Gasoline Release Site, Environmental Science \& Technology 36 , p. 5139-5146.

(11) Kuder T, Philp RP, Kolhatkar R, Wilson JT, Allen J (2003) Application of stable carbon and hydrogen isotopic techniques fot monitoring biodegradation of MTBE in the field. In NGWA/AP Petroleum Hydrocarbons and Organic Chenicals in Ground Water. American Petroleum Institute, Atlanta, USA

\section{Anschrift der Autoren:}

Michael Berg, Luc Zwank, Jakov Bolotin, Christoph Aeppli EAWAG (Eidg. Anstalt für Wasserversorgung, Abwasserreinigung und Gewässerscinutz)

Ueberlandstr: 133, CH-8600 Dübendorf, Scliweiz.

Tel. +41-1-823 5078, E-Mail: michael.berg@eawag.ch

Andreas Häner, Matthias Möller, Christoph Munz

BMG Engineering $A G$, Ifangstrasse 11, Cl-8952 Schlieren, Schweiz. www.brigeng.cl

Urs Ziegler

BUWAL. (Bundesamt für Umweit, Wald und Landschaft),

CH-3003 Bern, Schweiz. 